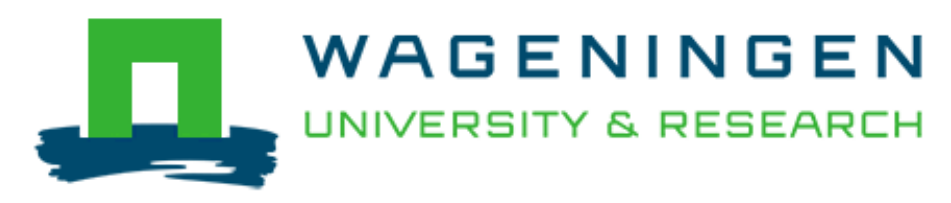

\author{
Fat Crystallite Thickness Distribution Based on SAXD Peak Shape Analysis \\ den Adel, R., van Malssen, K., van Duynhoven, J., Mykhaylyk, O. O., \& Voda, \\ A.
}

This article is made publically available in the institutional repository of Wageningen University and Research, under article 25fa of the Dutch Copyright Act, also known as the Amendment Taverne.

Article 25fa states that the author of a short scientific work funded either wholly or partially by Dutch public funds is entitled to make that work publicly available for no consideration following a reasonable period of time after the work was first published, provided that clear reference is made to the source of the first publication of the work.

For questions regarding the public availability of this article, please contact openscience.library@wur.nl.

Please cite this publication as follows:

den Adel, R., van Malssen, K., van Duynhoven, J., Mykhaylyk, O. O., \& Voda, A. (2018). Fat Crystallite Thickness Distribution Based on SAXD Peak Shape Analysis. European Journal of Lipid Science and Technology, 120(9), [1800222]. https://doi.org/10.1002/ejlt.201800222 


\title{
Fat Crystallite Thickness Distribution Based on SAXD Peak Shape Analysis
}

\author{
Ruud den Adel,* Kees van Malssen, John van Duynhoven, Oleksandr O. Mykhaylyk, \\ and Adrian Voda
}

Understanding of the multiscale structuring capability of triacylglycerols (TAGs) can be enhanced through knowledge of the crystallite thickness distribution (CTD), which can be obtained through X-ray diffraction peak shape analysis. The Fourier-transformation-based Bertaut-Warren-Averbach (BWA) method provides an approach to resolve CTDs in TAGs, where molecules are packed in repeating layers. The feasibility of using the BWA method is demonstrated for small angle X-ray diffraction (SAXD) measurements on both laboratory equipment and a synchrotron beamline for model TAG systems and real food products such as margarines.

Practical Applications: The CTDs enable discrimination of margarines produced by different processing routes and may be used as a signature of network structures that underlie product texture.

\section{Introduction}

Multiscale networks of triacylglycerol (TAG) crystallites critically determine the texture of fat-based products, like margarine, and butter. $^{[1]}$ The growth of these networks is governed by crystallization and aggregation, which can both be tuned by TAG composition and heat-cooling regimes. ${ }^{[2,3]}$ However, due to the strong coupling between crystallization and network formation, rational design of fat-based food products requires a multiscale view on the evolving network structure. For this purpose, X-ray scattering techniques are commonly used, since they can provide quantitative information on molecular packing, polymorphism, phase transitions ${ }^{[4]}$ crystallite thickness and organization in fractal networks. ${ }^{[5,6]}$ So far, the current suite of X-

R. den Adel, Dr. K. van Malssen, Prof. J. van Duynhoven, Dr. A. Voda Unilever R\&D Vlaardingen

Olivier van Noortlaan 120, 3130 AC Vlaardingen, The Netherlands

E-mail: ruud-den.adel@unilever.com

Prof. J. van Duynhoven

Laboratory of Biophysics

Wageningen University

Stippeneng 4, 6708 WE Wageningen, The Netherlands

Dr. O. O. Mykhaylyk

Soft Matter Analytical Laboratory

Department of Chemistry

The University of Sheffield

Sheffield S3 7HF, The United Kingdom

The ORCID identification number(s) for the author(s) of this article can be found under https://doi.org/10.1002/ejlt.201800222.

DOI: 10.1002/ejlt.201800222 ray techniques deployed in fats research was used to quantify only the average structural features obtained over different length scales. This is in particular the case for the TAG crystallite thickness, which can be considered as the smallest entity in multiscale networks. In this work, the challenge of quantitative assessment of the dispersion in crystallite thickness is addressed.

It is well-known that TAGs, due to their high aspect ratio caused by long acyl chains comprising the molecules, form crystal structures with disproportionate lattice parameters. This property of the TAG structures significantly complicates crystal structure analysis, due to both broadening of intensity range of diffraction peaks and increasing uncertainty of diffraction peaks indexing. ${ }^{[7]}$ However, the same property of the TAG structures gives an advantage for applications of other X-ray methods, especially techniques for measurements of crystallite size, and crystal lattice strain caused by structural imperfections. These methods are based on diffraction peak broadening and peak shape analysis. In particular, X-ray scattering patterns of TAGs consist of two sets of diffraction peaks: one set at small diffraction angles that corresponds to the longitudinal order of the TAGs packed in long period layers, and the other set, at wide angles of diffraction, corresponding to the transverse order of the molecules forming a small sub-cell. Thus, diffraction peaks possessing a common crystallographic direction $<001\rangle$ (i.e., $00 l$, where $l=1,2,3, \ldots$ is the peak order), originating from the TAG layers, are conveniently separated from the rest of the diffraction pattern. Moreover, the shape of each peak is virtually unaffected by the neighbors. Common TAG structures of two-acyl- (2L) or threeacyl- (3L) chain layer thickness, produce up to four or six distinct peak orders, respectively. ${ }^{[8-10]}$ Fat crystallites grow fast laterally and slow axially, which results in thin, long, and broad platelets. There is a reciprocal relationship between the crystallite thickness in each crystallographic direction and the broadening of corresponding diffraction peaks. With the use of small-angle X-ray diffraction (SAXD), the averaged crystallite thickness (ACT) of stacked TAG layers can be determined. In order to obtain information about the average thickness of fat crystallites (proportional to the number of repeating bi-layers of glycerides) the full width at half maximum (FWHM) value of the first order diffraction peak is commonly used. ${ }^{[1-3,5]}$ The SAXD region, where this first order diffraction peak typically appears, 
corresponds to a $d$-spacing between 7.0 and $3.5 \mathrm{~nm}\left(2 \theta=1.3^{\circ}\right.$ and $2.5^{\circ}$ for $\mathrm{CuK} \alpha$-radiation, respectively, where $\theta$ is the Bragg angle), depending on fat type and polymorph. The average crystallite thickness can be calculated using the Scherrer formula ${ }^{[11]}$.

$L=\frac{K \lambda}{\beta \cos \theta}$

where $\lambda$ is X-ray wavelength (in nm), $\beta$ is the FWHM in radians corrected for instrumental broadening and coefficient $K$ is $0.9 .^{[5]}$ The FWHM was calculated based on the width of the first order diffraction peak. For diffraction peaks corresponding to the layered structure of fat systems $\theta$ is small and, therefore, $\cos \theta$ can be approximated by unity. The Scherrer equation can be applied in a straightforward manner to assess ACTs, but does however not address the heterogeneity that can occur in crystallite thicknesses. Systems can have the same ACT, but very different crystallite thickness distribution (CTD), including a possible multi modal distribution.

The presence of up to six distinct peak orders makes TAG crystallites ideally suited for a rigorous $00 l$ peak broadening analysis by Fourier transformation-based techniques, in particular by the method developed by Bertaut ${ }^{[12]}$ and Warren and Averbach $^{[13]}$ (BWA). The results provided by Fourier methods include CTD and average lattice strain, all specified for the specific crystallographic direction. The BWA method was originally developed for metals, ${ }^{[13,14]}$ but the method is universal and can be applied to any crystalline material, provided certain experimental requirements are met. For example, the method was successfully adapted for measurements of domain thickness distribution of layered clay minerals where basal $00 \mathrm{l}$ reflections were used for recovering layer size distribution functions, including multimodal distribution of crystallite thickness. ${ }^{[15]}$ Considering the structural analogy between layered structures, the BWA method should also apply to TAGs. Since TAG crystallites are plate-like structures with molecules forming layers parallel to the flat surface, this provides the opportunity to obtain a TAG crystallite thickness distribution function, and not only the ACT. ${ }^{[11]}$

This study presents the proof of principle of using the BWA method to obtain the CTD in a fully saturated mono-acid triglyceride (tripalmitin), and in margarines produced by different processing routes and having a low or high amount of fat. The method will be applied on SAXD data acquired at a synchrotron source and on a labscale X-ray diffractometer.

\section{Experimental Section}

\subsection{Materials}

Tripalmitin ( $\geq 99$ wt\%) from Sigma Aldrich (The Netherlands) was used to validate the applicability of the BWA method to determine CTDs in fats. Tripalmitin was molten and subjected to fast and slow cooling conditions in order to manipulate the thickness of fat crystallites. The fast cooling experiment was performed by dripping small droplets of liquid tripalmitin from a glass pipette (outlet diameter $1 \mathrm{~mm}$ ) to a box filled with liquid nitrogen. The slow cooling experiment was performed by cooling from the melt to $20^{\circ} \mathrm{C}$ using a cooling rate of $5^{\circ} \mathrm{C} \mathrm{min}^{-1}$. Additionally, a fat crystal mixture (50-50 wt
\%) was made of these two tripalmitin samples. A tristearin standard $(\approx 99$ wt $\%$ ) from Sigma Aldrich (The Netherlands) was used for the instrumental peak broadening correction. The standard was cooled from the melt $\left(90^{\circ} \mathrm{C}\right)$ to $50^{\circ} \mathrm{C}$ at $1^{\circ} \mathrm{C} \mathrm{min}^{-1}$, subsequently heated to $60^{\circ} \mathrm{C}$ and kept isothermally for $60 \mathrm{~min}$ and then cooled again to $20^{\circ} \mathrm{C}$ at $1^{\circ} \mathrm{Cmin}^{-1}$. One fat spread was prepared using the conventional votator process, where water and a molten fat blend are rapidly cooled in a scraped surface heat-exchanger. ${ }^{[16]}$ Another spread was prepared by dispersing water in a slurry of micronized fat crystals (MFC) ${ }^{[17]}$ in oil. ${ }^{[18,19]}$ The fat blend composition and the total fat level in both spreads were identical. A set of 11 commercial spreads was obtained from local retailers. The percentage of water content in these spreads ranges from 25 to $66 \mathrm{wt} \%$.

\section{Methods}

\subsection{SAXD and WAXD Experiments}

SAXD and WAXD experiments were simultaneously performed at the high-brilliance ID02 beamline of the European Synchrotron Radiation Facility (ESRF) in Grenoble, France. The incident X-ray wavelength is $0.0996 \mathrm{~nm}$ for all experiments. The sample to detector distance is $1.50 \mathrm{~m}$ allowing collection of SAXD data in the range of $0.06<q\left(\mathrm{~nm}^{-1}\right)<4.5$ [or $104.7<d(\mathrm{~nm})<1.4$ ] and $0.1 \mathrm{~m}$ allowing collection of WAXD data in the range of $7.5<q$ $\left(\mathrm{nm}^{-1}\right)<51$ [or $0.8<d(\mathrm{~nm})<0.1$ ], where $q$ is the scattering vector modulus $[q=(4 \pi \sin \theta) / \lambda]$ and $d$ is the corresponding real space distance calculated from $2 \pi / q$. The samples are $2.0 \mathrm{~mm}$ thick and held in an aluminum cell with mica windows. A four-position temperature stage is used to control sample temperature at $10^{\circ} \mathrm{C}$. The SAXD detector is a FReLoN (Fast-Readout, Low-Noise) Kodak CCD based on a Kodak KAF-4320 image sensor and the WAXD detector a MCP-Sensicam CCD. The two-dimensional SAXD patterns are azimuthally averaged to obtain the scattered intensity as a function of $q$. A typical measurement time is $0.3 \mathrm{~s}$.

SAXD patterns were also measured on a laboratory scale Bruker D8 Discover X-ray powder diffractometer with a Våntec 500 2D detector and an I $\mu \mathrm{S}$ microfocus X-ray source $(\mathrm{CuKa}$ radiation, $\lambda=0.154184 \mathrm{~nm})$. The diffraction patterns were collected in a transmission mode with the X-ray beam perpendicular to the flat sample surface. A 1-mm collimating slit is used and the sample to detector distance is $32.5 \mathrm{~cm}$. A typical measurement time used for a fat spread is $900 \mathrm{~s}$. The SAXD patterns were collected from samples at $5^{\circ} \mathrm{C}$. A Linkam temperature stage (THMS600, Linkam scientific instruments, Tadworth, KT20 5LR, United Kingdom) is used to control the sample temperature. Flat samples are $2.0 \mathrm{~mm}$ thick and enclosed by X-ray Mylar film in the sample holder of this stage. 1D SAXD curves are obtained from the 2D patterns using a software supplied with the instrument.

\subsection{Crystal Thickness Distribution Calculations for Fats by a Method Based on Fourier Analysis}

Since there is an analogy between layered structures, a simplified Fourier analysis approach developed for basal reflections of clays based on the original works by Bertaut ${ }^{[12]}$ and Warren and 
Averbach, ${ }^{[13]}$ can be applied for CTD analysis of fats. For this purpose, the Mudmaster program ${ }^{[15,20]}$ is adapted, which has been proposed for studying coherent scattering domains and strain in minerals by the BWA method. This program includes the analysis of raw X-ray diffraction data, which makes it possible to extract the CTD from the peak shape. In summary, at the initial step, an XRD $00 l$ peak intensity distribution, where $l$ is the peak order, is corrected for the Lorentz-polarisation factor (LP), for a layer scattering factor (if required) and a scattering background. As a result of these corrections, the interference function of the peak $\phi_{00 l}\left(Z^{*}\right)$ expressed as a function of $Z^{*}(=2 \sin \theta / \lambda)$ is obtained, which contains information of the CTD and strain. At the next step the interference function is expanded in terms of a Fourier series:

$$
H(n)=\sum_{Z^{*}=\frac{2 \sin \left(\theta_{00 l}-\Delta \theta\right)}{\lambda}}^{\frac{2 \sin \left(\theta_{00 l}+\Delta \theta\right)}{\lambda}} \phi_{00 l}\left(Z^{*}\right) \cos \left(2 \pi Z_{n} Z^{*}\right)
$$

where $Z_{n}=n d(001), n$ is the number of fat molecule structural layers, $d(001)$ is the interlayer spacing, $\theta_{00 l}$ is the XRD peak position, $2 \Delta \theta$ is the width of analyzed Bragg angle interval. The Fourier coefficients calculated from Equation (2) are related to the CTD function $f(M)$, represented via a number of TAG layers in the crystallites, $M$ :

$$
H(n)=\frac{1}{\bar{M}} \sum_{M=M_{1}}^{M_{2}}(M-n) f(M)
$$

where $M_{1}$ and $M_{2}$ correspond to CTDs having the smallest and the largest number of TAG layers, respectively, and $\bar{M}$ is the mean number of layers defined as:

$$
\bar{M}=\sum_{M=M_{1}}^{M_{2}} M f(M) \text { and } \sum_{M=M_{1}}^{M_{2}} f(M)=1
$$

It follows from Equation (3) that the mean thickness (ACT) and the thickness distribution (CTD) can be obtained from $H(n)$ :

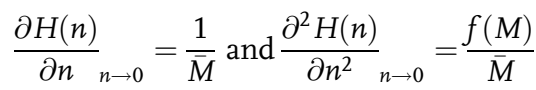

The ACT and CTD can be obtained from $\bar{T}=\bar{M} d(001)$ and $f$ $(T)$, respectively. It has to be noted that the Fourier coefficients calculated from the interference function [Equation (2)] may require corrections for instrumental broadening and symmetrical strain. Furthermore, the CTD obtained from the analysis may require smoothing and truncation to eliminate noise. For the comparison of multiple distributions and for visual inspection a connected dot graphing representation was chosen.

\section{Results and Discussion}

\subsection{Crystal Thickness Distribution Calculations}

Well-ordered TAG crystallites produce a set of diffraction peaks at small scattering angles associated with a layered packing of fat molecules. However, fat systems typically produce only a relatively strong first order diffraction peak corresponding to a layered structure with vanishingly weak higher order peaks. For this reason, the focus is on the shape analysis of the first order diffraction peak. The $2 \theta$-dependence of SAXD peak intensity can be distorted by both the instrument geometry and the X-ray beam polarization used for the data collection as well as by structural imperfections caused by molecule packing along the layer normal. Considering the fact that the first order diffraction peak intensity (or the corresponding structural amplitude) originating from 2L layers of the studied fats is virtually unaffected by possible structural imperfections such as movements of molecules and change of their position along the layer normal, ${ }^{[8]}$ correction for $2 \theta$-dependence of the layer scattering intensity is not required. Since $2 \theta$ position of the peak maximum is at a relatively small angle (between 1.3 and $2.5^{\circ}$ ) where changes of the polarization factor are negligible (about $0.3 \%{ }^{[21]}$ ), also correction of the peak shape distorted by $2 \theta$-dependence of the polarization factor is unnecessary. Furthermore, it has been found out that the Lorentz factor, demonstrating a relatively strong $2 \theta$-dependence at small angles, has a little effect on the Fourier transformation results. ${ }^{[21]}$ Thus, neither LP factor and layer scattering factor corrections of the diffraction peak needed to be performed before the peak broadening analysis. After removing the scattering background, the interference function $\phi_{001}\left(Z^{*}\right)$ is obtained, which contains information about CTD and strain. Size effects are independent of the diffraction peak order, whereas strain effects vary with the peak order. Thus, at least two o0l XRD peaks are needed to count structural strain in the BWA analysis. ${ }^{[13,14]}$ It can be demonstrated by a Williamson-Hall plot ${ }^{[22]}$ that the contribution of strain to 001 peak broadening of fats is significantly less than to the higher order peaks. A Williamson-Hall analysis of the high-quality synchrotron measurements of tripalmitin, processed at different crystallization conditions (Figure S1, Supporting Information), shows that the contribution of strain is less than $10 \%$ based on the fitting line slope (Figure S2, Supporting Information). Therefore, the peak shape of the 001 reflection can be assumed to be mostly determined by crystallite size, and no strain correction is required. This is a useful estimation, since in the case of low concentration samples such as fats in a spread product, the higher order reflections have a too low intensity to be analyzed.

\subsection{Performance of CTD Assessment Using Synchrotron SAXD Data}

The performance of the CTD analysis and its applicability to fats was first investigated using synchrotron SAXD data. Fast and slow cooled tripalmitin and a mixture of these samples were measured at the ID02 beamline at the ESRF, and SAXD patterns of the first order long spacings are clearly different with respect to linewidths at half heights and peak shapes (Figure S1, Supporting Information).

In Figure 1, CTDs of tripalmitin obtained by BWA analysis of the 001 peaks are represented as the $f(T)$ function plotted against the thickness of TAG crystallites, T. For pure tripalmitin, one bilayer $[d(001)]$ corresponds to $4.5 \mathrm{~nm}$. Thus, the volume weighted $f(T)$ [Equation (4)] represents a normalized frequency 


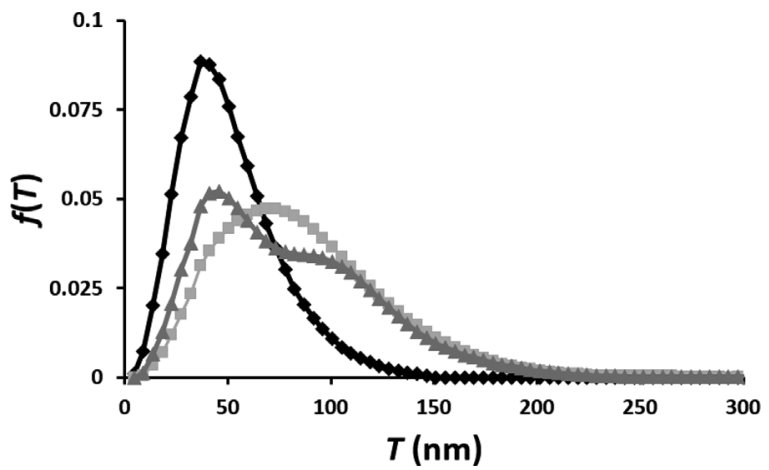

Figure 1. Volume weighted crystallite thickness distribution curves, $f(T)$, extracted from BWA analysis of 001 diffraction peaks of tripalmitin crystallized from the melt at different thermal conditions: Fast cooled (black diamonds), slow cooled (light gray squares) and a 50:50wt\% mixture thereof (gray triangles).

distribution histogram, composed of integer numbers of bilayers. The volume weighted mean thicknesses, $\bar{T}$, for fast and slowly cooled tripalmitin are respectively 54 and $86 \mathrm{~nm}$. This is in line with the ACTs obtained by the Scherrer approach [Equation (1)] which are respectively 59 and $96 \mathrm{~nm}$. Figure 1 shows that the width of the CTD for the slowly cooled tripalmitin is much broader than for the fast cooled one, indicating less uniformity in crystallite thicknesses. Both CTDs are asymmetrical, reminding a log normal distribution with a "tailing" effect toward higher thicknesses. The CTD obtained for the mixture of fast and slow cooled tripalmitin resembles the superposition CTDs of its constituents (Figure 1), which validates that bimodal populations can accurately be determined by the BWA method. Since WAXD measurements showed the a polymorph for all tripalmitin samples, the obtained CTDs are generated by the cooling process and not the result of a polymorphic transition.

\subsection{Performance of CTD Assessment Using Laboratory Instrument SAXD Data}

In contrast to synchrotron SAXD measurements, data acquired on a laboratory instrument require a correction during CTD analysis to accommodate instrumental line broadening. To establish the feasibility using the method in combination with a laboratory instrument, CTDs of a commercial spread were obtained using SAXD data obtained from both a synchrotron beamline and a laboratory instrument (Figure 2). In first instance (Figure 2, the left graph) no correction for instrumental broadening was applied for the laboratory SAXD data. Since in that case line broadening is not accounted for, this will result in an overestimation of small crystallites and an underestimation of larger ones. Indeed, a small systematic deviation can be observed between the uncorrected CTD obtained on the laboratory instrument and the CTD obtained using a synchrotron beamline (Figure 2, the left graph). Hence, for obtaining accurate CTDs, correction for the instrumental broadening of labscale equipment, by using a standard (tristearin) is recommended. This standard was measured under the same conditions as the spread samples, and the first order long spacing was used for the BWA analysis. The instrumental broadening was removed from the Fourier coefficients by using the Stokes equation. ${ }^{[23]}$ The resulting CTD of the commercial spread is virtually overlapping with the one obtained from synchrotron measurements (Figure 2, the right graph), demonstrating the effectiveness of the correction procedure.

\subsection{Recognition of Margarine Processing Signatures}

In order to assess the impact of industrial processing conditions on CTDs, measurements were performed on two margarines with identical fat composition but manufactured by either the conventional votator process, or by direct emulsification in a micronized fat crystal (MFC) dispersion. ${ }^{[19]}$ In comparison with the votator process, the MFC process allows manufacturing of stable emulsions with a smaller amount saturated fat. No significant differences in the presences of the $\beta^{\prime}$ and $\beta$ polymorphs were found. The ACT values derived from the Scherrer equation Equation (1) are 30 and $44 \mathrm{~nm}$, for respectively the votator and MFC processed margarines. This is in line with the volume weighted mean thicknesses obtained from CTDs, which are 33 and $41 \mathrm{~nm}$. The CTDs shown in Figure 3 show further distinct differences in the shape of the distributions. Whereas the votator-processed margarine has an almost symmetric distribution of thin crystallites, the one for the MFC-processed margarine is skewed toward thicker ones. In
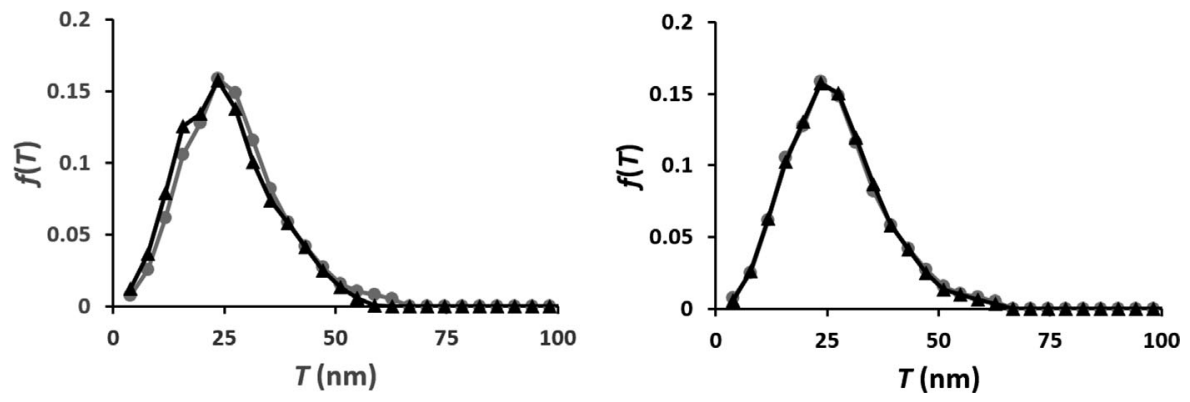

Figure 2. Comparison of volume weighted CTD curves, $f(T)$, extracted from BWA analysis of 001 diffraction peak of a votator low-fat spread measured by a laboratory diffractometer (black triangles) and at a synchrotron beamline (ESRF, ID02) (gray circles). The left and the right graph represents CTD curves obtained without and with the instrumental broadening correction for the laboratory diffractometer data, respectively. 


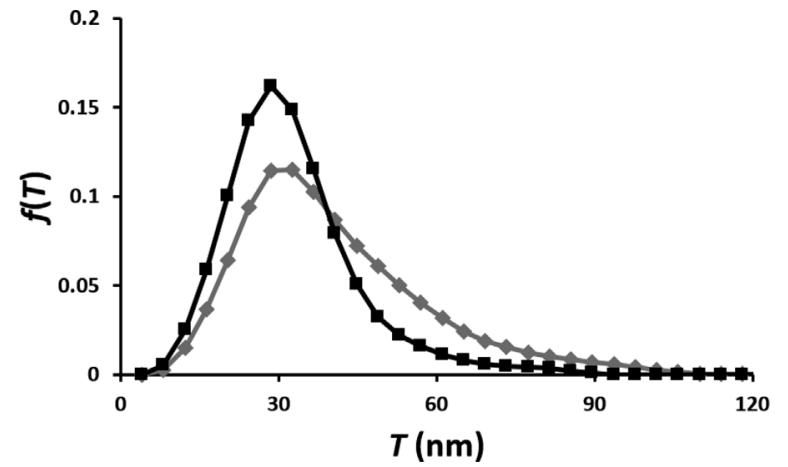

Figure 3. Volume weighted crystallite thickness distribution curves, $f(T)$, extracted from BWA analysis of 001 diffraction peak of $34 \%$ fat spreads manufactured by means of the MFC (gray diamonds) and votator (black squares) processes.

order to investigate whether the CTD could be exploited for recognizing industrial manufacturing routes, a set of 11 commercial margarines, produced by either votator or MFC processing, was measured at the synchrotron beamline (Figure 4). Of note, the formulation (TAG composition, emulsifiers, percentage of the water fraction) of these commercial products varied widely. The margarine samples showed the presence of both $\beta$ ' polymorph and $\beta$.

In Figure 4, two types of CTDs could be recognized: a near symmetric distribution around $25 \mathrm{~nm}$ or a distribution around $32 \mathrm{~nm}$, skewed toward thicker crystallites. All votator low-fat spreads are in the symmetric CTD group, whereas the MFC lowfat spreads are in the same group as the votator high fat group. This is in line with the high textural quality being produced by both the high fat votator process and the low fat MFC process. In contrast, the symmetric CTD seen for margarines manufactured by the low fat votator process corresponds to a poor textural quality. Hence, the CTD provides a signature for the quality of crystallite network structure, which was not confounded by the broad formulation range of the commercial products.

In this work, the CTD is presented as a structural feature that can be used to recognize the textural quality of a fat-based product. This suggests that the CTD is a main determinant of

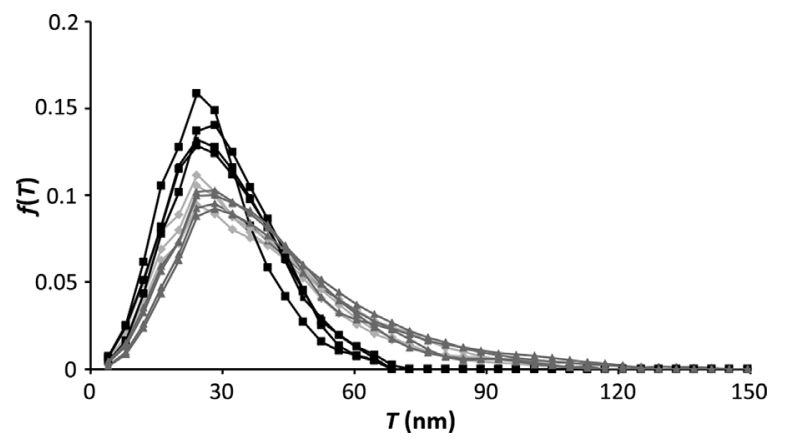

Figure 4. Volume weighted CTD curves, $f(T)$, extracted from BWA analysis of 001 diffraction peaks of various commercial spreads with low fat content obtained by the MFC (light gray diamonds) and votator process (black squares) and with a high fat content obtained by votator process (gray triangles). the arrangement of crystallites in multiscale network structures. It is tempting to attribute the skewed distribution to the different requirements of crystallite thickness for stabilization of the water droplet interface and the continuous fat phase. Whether and how the CTD can be tuned by formulation and processing, and how this structural feature determines recrystallization at droplet interface and in the continuous phases rewards further research.

\section{Conclusions}

It has been demonstrated that the CTD of fat crystallites can be obtained by analyzing SAXD patterns of the 001 diffraction peak of TAGs by using the BWA method. The method requires SAXD measurements with a good signal to noise ratio, sufficient baseline on either side of the diffraction peak and a sufficient resolution of the peak from neighboring peaks. Since SAXD data obtained using a synchrotron beamline are virtually free of instrumental aberrations, they can be used for direct assessment of the CTD of fats, irrespective of the type of polymorph present. After correction for instrumental line broadening, also laboratory-scale equipment can provide accurate CTDs. The BWA method was shown to be sensitive to shifts in CTD induced by tempering regimes, and also able to accurately assess a bimodal CTD. Furthermore, it was recognized that in food products such as margarines the CTD can be used as a signature of a network structure that relates to product performance. Further research should focus on how processing and formulation can steer the CTD, and ultimately how it can be related to crystallite network structure and product texture.

\section{Abbreviations}

ACT, average crystal thickness; BWA, Bertaut-Warren-Averbach; CTD, crystal thickness distribution; ESRF, European Synchrotron radiation facility, FWHM, full width at half maximum; MFC, Micronized fat crystals; TAGs, Triacylglycerols; SAXD, small angle X-ray diffraction; WAXD, wide angle X-ray diffraction.

\section{Supporting Information}

Supporting Information is available from the Wiley Online Library or from the author.

\section{Acknowledgments}

The authors would like to thank T. Narayanan and M. Sztucki (ESRF) for support during experiments on the ID02 beamline in Grenoble. Jan Wieringa, Jo Janssen, Michiel Meeuse and Peter de Groot from Unilever Discover Vlaardingen are greatly acknowledged for fruitful discussions.

\section{Conflict of Interest}

R.d.A., J.v.D., K.v.M., and A.V. are employed by a company that manufactures and markets lipid-based food products. 


\section{ADVANCED \\ SCIENCE NEWS}

www.advancedsciencenews.com

\section{Keywords}

Bertaut-Warren-Averbach analysis, crystallite thickness distribution, triacylglycerol, margarine, small angle $\mathrm{X}$-ray diffraction

Received: May 9, 2018

Revised: July 4, 2018

Published online: August 1, 2018

[1] N. C. Acevedo, A. G. Marangoni, Cryst. Growth Des. 2010, 10, 3327.

[2] N. C. Acevedo, A. G. Marangoni, Cryst. Growth Des. 2010, 10, 3334.

[3] N. C. Acevedo, F. Peyronel, A. G. Marangoni, Curr. Opin. Colloid Interface Sci. 2011, 16, 374.

[4] N. Garti, K. Sato, Crystallization Processes in Fats and Lipids Systems. Marcel Dekker, New York 2001.

[5] N. C. Acevedo, A. G. Marangoni, Annu Rev Food Sci Technol. 2015, 6, 71.

[6] F. Peyronel, B. Quinn, A. G. Marangoni, J. Phys. Condens. Matter. 2014, 26, 464110.

[7] A. van Langevelde, R. Peschar, H. Schenk, Acta Crystallographica Section B 2001, 57, 372.

[8] O. O. Mykhaylyk, I. W. Hamley, J. Phys. Chem. B 2004, 108, 8069. www.ejlst.com

\section{Lipid Science and Technology}

[9] R. Peschar, H. Schenk, J. B. van Mechelen, Acta Crystallographica Section B 2006, 62, 1121.

[10] A. van Langevelde, K. van Malssen, F. Hollander, R. Peschar, H. Schenk, Acta Crystallographica Section B 1999, 55, 114.

[11] P. Scherrer, Göttinger Nachrichten Gesell. 1918, 2, 98.

[12] M. E. Bertaut, Acta Crystallogr. 1950, 3, 14.

[13] B. E. Warren, B. L. Averbach, J. Appl. Phys. 1950, 21, 595.

[14] B. E. Warren, B. L. Averbach, J. Appl. Phys. 1952, 23, 497.

[15] V. A. Drits, D. D. Eberl, J. Srodon, Clays Clay Miner. 1998, 46, 38.

[16] A. Bot, E. Flöter, Edible Oil Processing, Second Edition. WileyBlackwell, Chichester, UK 2013, Chapter 8.5, 234.

[17] P. Munuklu, P. J. Jansens, J. Supercrit. Fluids 2007, 40, 433.

[18] B. J. Arends, C. M. Beindorff, A. J. Bezemer, T. de Man, European Patent WO2013079279 A1 (2013).

[19] T. Nikolaeva, R. den Adel, E. Velichko, W. G. Bouwman, D. HermidaMerino, H. Van As, A. Voda, J. van Duynhoven, Food and Function 2018, 9, 2102.

[20] D.D. Eberl, V.A. Drits, J. Scrodon, R. Nüesch, U. S. Geological Survey Open File Report 1996, 96 revised 8/24/00.

[21] T. Unruh, H. Bunjes, K. Westesen, M. H. J. Koch, Colloid. Polym. Sci. 2001, 279, 398.

[22] G. K. Williamson, W. H. Hall, Acta Metall. 1953, 1, 22.

[23] H. P. Klug, L. E. Alexander, X-ray Diffraction Procedures for Polycrystalline and Amorphous Materials, second edition. John P. Wiley and Sons, Inc, N.Y. 1974, p. 966. 